\title{
Rayleigh Scattering Diagnostic for Measurement of Temperature, Velocity, and Density Fluctuation Spectra
}

\author{
Amy F. Mielke* \\ NASA Glenn Research Center, Cleveland, OH, 44135 \\ Kristie A. Elam ${ }^{\dagger}$ \\ Jacobs Sverdrup, Cleveland, $\mathrm{OH}, 44135$ \\ and \\ Chih-Jen Sung ${ }^{\ddagger}$ \\ Case Western Reserve University, Cleveland, OH, 44106
}

\begin{abstract}
A molecular Rayleigh scattering technique is developed to measure dynamic gas temperature, velocity, and density in unseeded turbulent flows at sampling rates up to 10 kHz. A high power $\mathrm{CW}$ laser beam is focused at a point in a heated air jet plume and Rayleigh scattered light is collected and spectrally resolved. The spectrum of the light, which contains information about the temperature, velocity, and density of the flow, is analyzed using a Fabry-Perot interferometer. The circular interference fringe pattern is divided into four concentric regions and sampled at 1 and $10 \mathrm{kHz}$ using photon counting electronics. Monitoring the relative change in intensity within each region allows for measurement of gas temperature and velocity. Independently monitoring the total scattered light intensity provides a measure of gas density. Power spectral density calculations of temperature, velocity, and density fluctuations, as well as mean and fluctuating quantities are demonstrated for various radial locations in the jet flow at a fixed axial distance from the jet exit plane. Results are compared with constant current anemometry and pitot probe measurements at the same locations.
\end{abstract}

\section{Nomenclature}

$=$ most probable molecular speed $\left(\mathrm{m} \mathrm{s}^{-1}\right)$

$=$ speed of light $\left(=2.998 \times 10^{8} \mathrm{~m} \mathrm{~s}^{-1}\right)$

$=$ Fabry-Perot mirror spacing $(\mathrm{m})$

$=$ incident electric field vector $\left(\mathrm{N} \mathrm{C}^{-1}\right)$

$=$ Fabry-Perot instrument function contrast

$=$ frequency of scattered light $\left(\mathrm{s}^{-1}\right)$

$=$ frequency of incident laser light $\left(\mathrm{s}^{-1}\right)$

$=$ collimating lens focal length $(\mathrm{m})$

$=$ fringe forming lens focal length $(\mathrm{m})$

$=$ sampling rate $\left(\mathrm{s}^{-1}\right)$

$=$ Planck's constant $\left(=6.626 \times 10^{-34} \mathrm{~N} \mathrm{~m} \mathrm{~s}\right)$

$=$ Fabry-Perot instrument function

$=$ interaction wave vector $\left(\mathrm{m}^{-1}\right)$

$=$ magnitude of $\mathbf{K}\left(\mathrm{m}^{-1}\right)$

$=$ incident light wave vector $\left(\mathrm{m}^{-1}\right)$

$=$ scattered light wave vector $\left(\mathrm{m}^{-1}\right)$

\footnotetext{
${ }^{*}$ Research Engineer, Optical Instrumentation and NDE Branch, Member AIAA

'Optics Technician

* Associate Professor, Department of Mechanical and Aerospace Engineering, Senior Member AIAA
} 


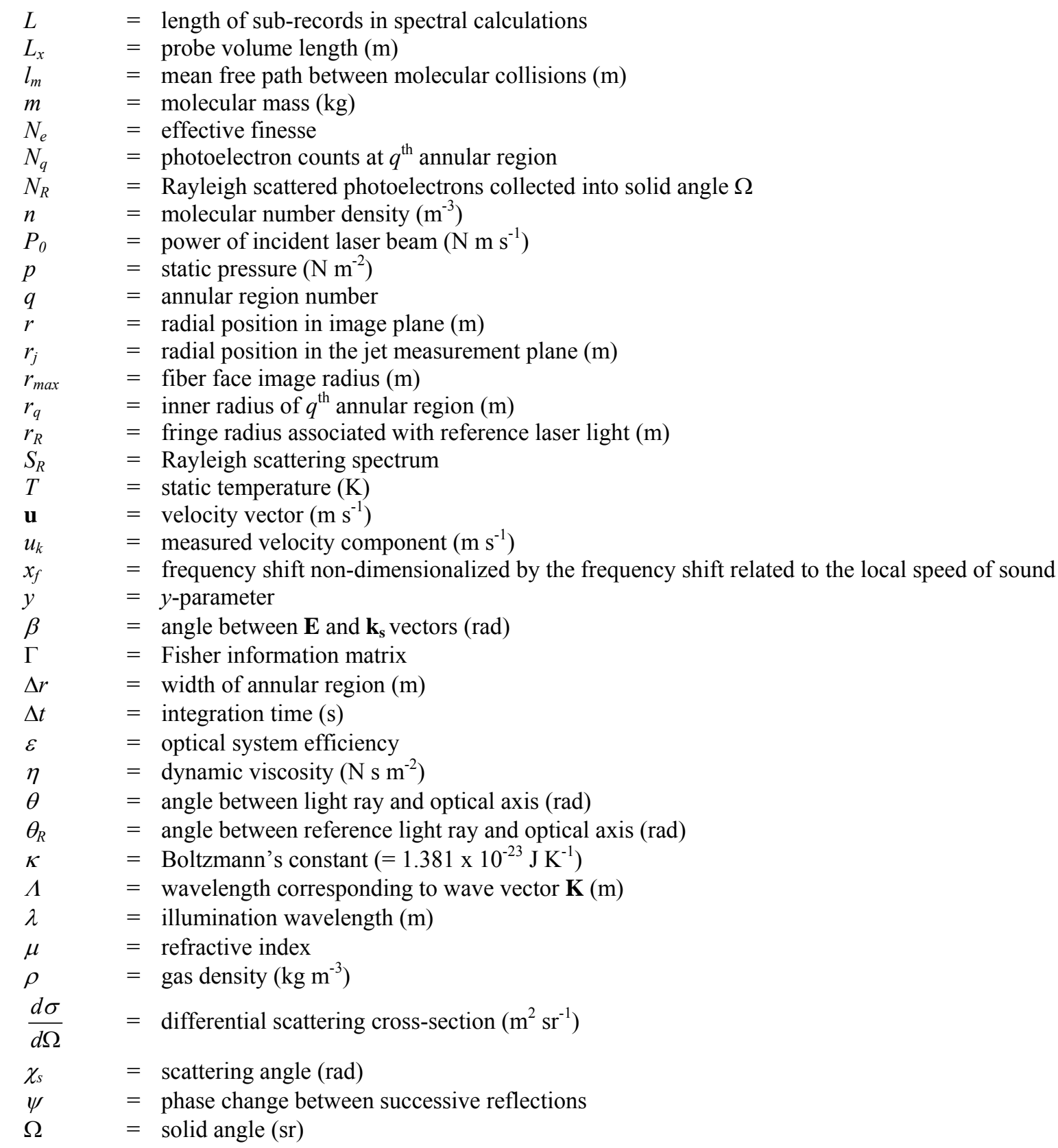

\section{Introduction}

$\mathrm{T}$ here is currently no high frequency response, non-intrusive temperature measurement technique for use in turbulent flow studies. There are also no techniques that can provide non-intrusive dynamic measurement of gas temperature, velocity, and density simultaneously. Conventional intrusive measurement devices such as resistance wires, pressure probes, and thermocouples are usually limited in spatial and temporal response, disturb the flow under study, and can be damaged by high pressure or temperature. Therefore, a non-intrusive molecular Rayleigh scattering technique is developed to measure dynamic gas temperature, velocity, and density in unseeded turbulent flows at sampling rates up to $10 \mathrm{kHz}$.

Various optical techniques are available for temperature, molecular number density, and velocity measurements. Eckbreth $^{1}$ provides the details of several techniques, such as Coherent Anti-Stokes Raman Spectroscopy (CARS), Spontaneous Raman and Rayleigh scattering, and Laser-Induced Fluorescence (LIF), with an emphasis on their use in combustion applications. Other techniques that have been used for flow measurements in various environments include Laser-Induced Thermal Acoustics (LITA) $)^{2,3}$, also known as Transient Grating Spectroscopy (TGS) ${ }^{4}$, Laser- 
Doppler Velocimetry (LDV) ${ }^{5-8}$, filtered Rayleigh scattering ${ }^{9}$, Particle Image Velocimetry (PIV) ${ }^{10}$, and focusing schlieren deflectometry ${ }^{11}$. However, none of these techniques can provide high frequency response measurement of all three flow properties simultaneously.

The temporal response of most of the optical measurement techniques mentioned here is typically limited by the repetition rate of pulsed lasers, usually on the order of $10 \mathrm{~Hz}$; however one can potentially take many snap shots (1-5 ns temporal resolution) to obtain turbulence measurements. Signal strength of the scattering process can also limit the temporal response. Rayleigh scattering has several advantages over other light scattering techniques. The Rayleigh scattering cross-section is three orders of magnitude greater than the Raman scattering cross-section, and Rayleigh scattering is generally much simpler to implement than techniques that require multiple lasers and have other complicating factors.

Previous works using molecular Rayleigh scattering to make temperature, velocity, and number density measurements in harsh environments have been reported ${ }^{12-15}$. The current work is an extension of a previously developed technique using Rayleigh scattering to obtain dynamic density and velocity measurements in supersonic free jets ${ }^{16,17}$ and dynamic density and temperature measurements in heated jets ${ }^{18}$. The Rayleigh scattering technique presented is unique in that it provides simultaneous temperature, velocity, and density fluctuation measurements. A high power CW laser beam is focused at a point in a jet plume and Rayleigh scattered light is collected and spectrally resolved. The spectrum of the light contains information about the temperature, velocity, and density of the flow. A planar mirror Fabry-Perot interferometer (FPI) is used to analyze the spectrum of the scattered light, and photomultiplier tubes (PMTs) record the fringe intensity pattern at high sampling rates enabling dynamic measurement of the gas flow properties.

The ability to obtain high frequency response measurement of multiple properties simultaneously is a valuable tool for compressible, turbulent flow research. These data are particularly useful to aeroacoustics researchers who are interested in correlating flow property fluctuations with far field acoustic fluctuations. Knowledge of velocitytemperature fluctuation correlations is also needed to improve computational fluid dynamic (CFD) models of compressible turbulent flows.

\section{Theory}

\section{A. Rayleigh scattering and spectral analysis}

Molecular Rayleigh scattering is the result of elastic light scattering from gas molecules. When light from a single frequency laser beam passes through a gas, the scattered light is shifted in frequency by the Doppler effect due to the thermal as well as the bulk motion of the molecules. The frequency spectrum of the scattered light contains information about the gas density, bulk velocity, and temperature. Figure 1 shows a Rayleigh scattering spectrum containing the narrow laser line and a typical Rayleigh spectral peak to illustrate how the flow property measurements are obtained from the spectral information. If the gas composition is fixed, the total intensity of the Rayleigh scattered light is directly proportional to the gas density. The frequency shift between the laser peak and the Rayleigh peak is proportional to the bulk flow velocity. The width of the spectrum is related to the gas temperature.

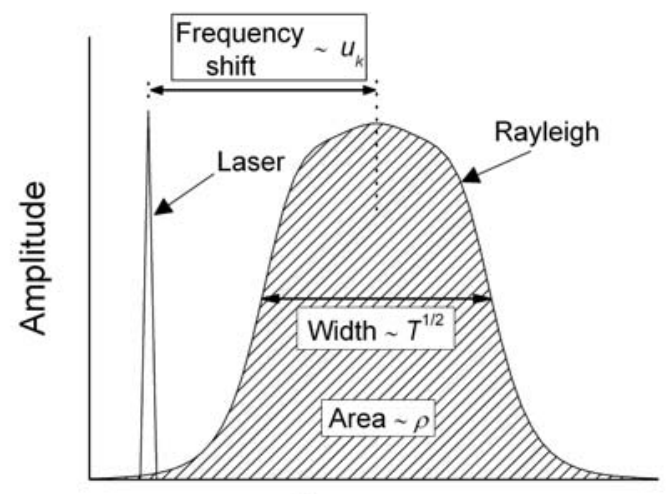

Frequency

Figure 1. Rayleigh scattering spectrum.

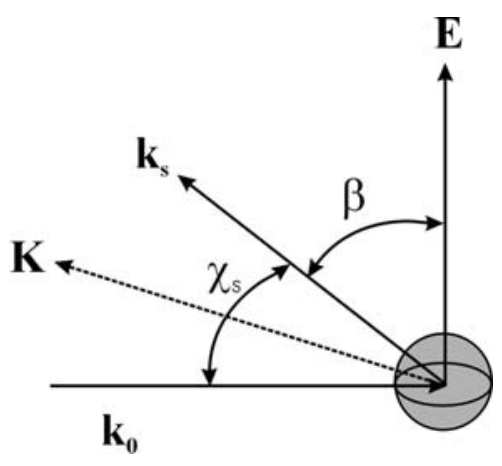

Figure 2. Light scattering from a moving particle. 
The shape of the spectrum is dependent on gas pressure, temperature and the scattering angle ${ }^{19}$. A nondimensional parameter $y$, which represents the ratio of the wavelength of the scattering grating $\left(\Lambda=\frac{\lambda}{2 \sin \frac{\chi_{s}}{2}}\right)$ to the mean free path between molecular collisions $\left(l_{m}=\frac{n \kappa T}{a \eta}\right)$, is used to establish spectral shape regimes:

$$
y=\frac{\Lambda}{2 \pi l_{m}}=\frac{p}{\eta K a}
$$

where

$$
a=\sqrt{\frac{2 \kappa T}{m}}
$$

The interaction wave vector, which defines the direction of the velocity component being measured, is the bisector of the incident and scattered light wave vectors (Fig. 2). The interaction wave vector and its magnitude are given by:

$$
\begin{gathered}
\mathbf{K}=\mathbf{k}_{\mathbf{s}}-\mathbf{k}_{\mathbf{0}} \\
K=|\mathbf{K}|=\frac{4 \pi}{\lambda}\left[\sin \frac{\chi_{s}}{2}\right]
\end{gathered}
$$

The geometry of the optical arrangement in an experiment can be designed such that the desired component of the velocity is measured:

$$
u_{k}=\frac{\mathbf{K} \cdot \mathbf{u}}{K}
$$

Three spectral shape regimes are defined for typical $90^{\circ}$ scattering $^{19-21}$. For low density gases where $y<<1$, the Rayleigh spectrum is accurately modeled by a Gaussian function and the gas is said to be in the Knudsen or collisionless regime. For higher density gases where $y \gg>1$, the Rayleigh spectrum broadens and eventually develops side-lobes known as Brillouin peaks. This is known as the hydrodynamic or continuum regime where molecular collisions are the dominant process. Finally, for $0.2 \leq y \leq 2$, as is the case in the present experiments, the gas is in a transition region between the collisionless and hydrodynamic regimes, and a kinetic theory model is required. The shape regimes are illustrated in Fig. 3, where the normalized frequency is defined as:

$$
x_{f}=\frac{2 \pi\left(f-f_{0}\right)}{K a}
$$

A kinetic theory model developed by $\mathrm{G}$. Tent ${ }^{19,22}$ provides a description of Rayleigh-Brillouin scattering from molecular gases in all density regimes. The TENTI S6 spectrum model ${ }^{19,22}$ was used to generate the information displayed in Figs. 1 and 3, and is incorporated in the model function used in least squares analysis of the experimental data.

The spectrum of the Rayleigh scattered light is analyzed using a planar mirror FPI (Fig. 4) operated in the static imaging mode ${ }^{23}$. The fringe intensity pattern is a function of the Rayleigh spectrum and the Fabry-Perot instrument function. The Fabry-Perot instrument function is:

$$
I_{F P}\left(x_{f}, r\right)=\frac{1}{1+F \sin ^{2} \frac{\psi\left(x_{f}, r\right)}{2}}
$$


where

$$
\begin{gathered}
F=\frac{1}{\sin ^{2}\left(\frac{\pi}{2 N_{e}}\right)} \\
\psi=\frac{4 \pi \mu d}{\lambda}\left[\frac{\theta_{R}^{2}-\theta^{2}}{2}+\frac{\lambda a}{c \Lambda} x_{f}\right] \\
\theta=r / f_{L}
\end{gathered}
$$

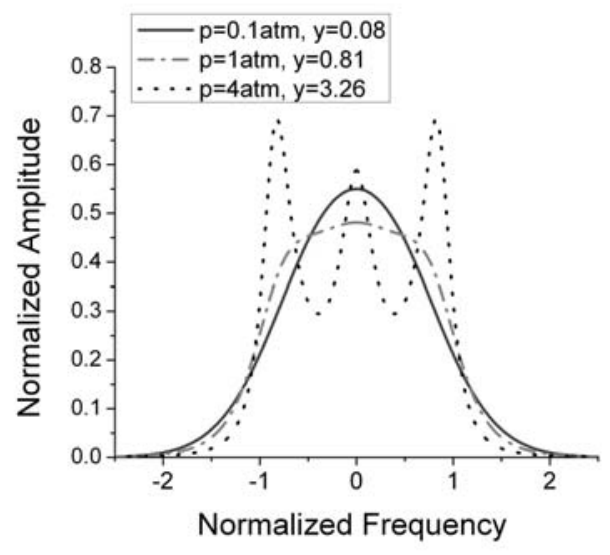

Figure 3. Rayleigh scattering spectrum for various $y$-parameters.

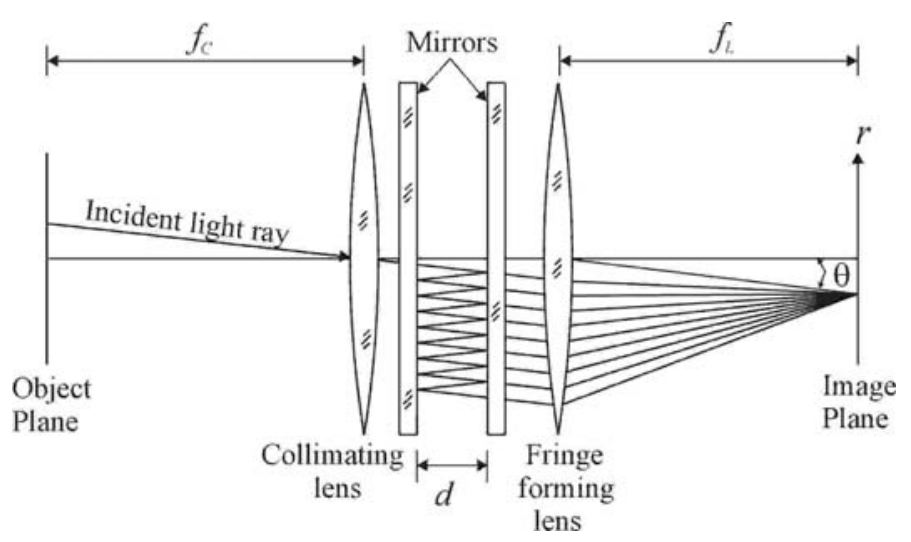

Figure 4. Fabry-Perot Interferometer.

In our experiment, Rayleigh scattered light from a defined probe volume is collected into a multimode optical fiber. The fiber directs the light to the FPI and a lens at the interferometer output focuses the interference fringe pattern at the image plane. The total expected number of Rayleigh photoelectron counts without the FPI in the optical path can be expressed as:

$$
\left\langle N_{R}\right\rangle=\frac{\varepsilon P_{0} n L_{x} \lambda \Omega \Delta t}{h c}\left(\frac{d \sigma}{d \Omega}\right) \sin ^{2} \beta
$$

where the overall system efficiency $\varepsilon$ includes detector quantum efficiency and other losses. When the interferometer is placed in the light path, the model function for the amount of energy collected from the $q^{\text {th }}$ annular region of the interference pattern, which has been dissected into one circular and three annular regions, in terms of photoelectron counts, can be expressed as follows:

$$
\left\langle N_{q}\right\rangle=\frac{\left\langle N_{R}\right\rangle^{r_{q}+\Delta r}}{\pi r_{\max }^{2}} \int_{r_{q}}^{\infty} \int_{-\infty}^{\infty} S_{R}\left(x_{f}\right) I_{F P}\left(x_{f}, r\right) d x_{f} d r
$$

where the Rayleigh spectrum is evaluated using the TENTI S6 model.

\section{B. Lower bound for measurement uncertainty}

The lower bound on the uncertainty in temperature, velocity, and density measurements using Rayleigh scattering is set by the photon statistical (shot) noise. Estimates of the measurement uncertainty in the unknown parameters for this technique were obtained by Cramer-Rao lower bound analysis ${ }^{24}$. For a measurement that is a function of a set of unknown parameters, $\hat{\alpha}_{i}$, the variance of the estimates of the parameters is: 


$$
V\left(\hat{\alpha}_{i}\right)=\left\lfloor\Gamma^{-1}\right\rfloor_{i i}
$$

where the Fisher information matrix for Poisson statistics is given by:

$$
\Gamma_{i, j}=\sum_{q} \frac{1}{\left\langle N_{q}\right\rangle} \frac{\partial\left\langle N_{q}\right\rangle}{\partial \alpha_{i}} \frac{\partial\left\langle N_{q}\right\rangle}{\partial \alpha_{j}}
$$

Using the model function developed for $\left\langle N_{q}\right\rangle$, the uncertainty or standard deviation $\sigma\left(\hat{\alpha}_{i}\right)$ in each unknown parameter may be determined by calculation and inversion of the Fisher information matrix.

If a Gaussian spectrum is assumed, the lower bounds for uncertainties in temperature $T$, velocity $u_{k}$, and density $\rho$ for an ideal instrument are:

$$
\begin{aligned}
& \frac{\sigma(T)}{T}=\left(\frac{2}{\left\langle N_{R}\right\rangle}\right)^{1 / 2} \\
& \frac{\sigma\left(u_{k}\right)}{a}=\left(\frac{1}{2\left\langle N_{R}\right\rangle}\right)^{1 / 2} \\
& \frac{\sigma(\rho)}{\rho}=\left(\frac{1}{\left\langle N_{R}\right\rangle}\right)^{1 / 2}
\end{aligned}
$$

For air flow at $T=360 \mathrm{~K}, u_{k}=9 \mathrm{~m} / \mathrm{s}$, and standard pressure, the lower bound uncertainty estimates for a sampling rate of $10 \mathrm{kHz}$, assuming an ideal instrument, are:

$$
\frac{\sigma(T)}{T}=3.6 \% \quad \sigma\left(u_{k}\right)=8 \mathrm{~m} / \mathrm{s} \quad \frac{\sigma(\rho)}{\rho}=5.4 \%
$$

For any real instrument, these measurement uncertainties will be higher. The actual Cramer-Rao lower bound measurement uncertainties were calculated, given the Fabry-Perot interferometer, PMTs, and overall efficiency of the current system. The TENTI S6 spectral model ${ }^{19,22}$ was used to calculate the Rayleigh scattering spectrum in this analysis. Figure 5 shows the uncertainty in $T$ and $u_{k}$ over a range of fringe radii $\left(r_{R}\right)$ related to the incident (reference) laser light. This analysis was used to determine the reference fringe radius resulting in minimum uncertainty levels in both $T$ and $u_{k}$. A $r_{R}$ value of $6.5 \mathrm{~mm}$ was chosen for the experiments. Figure 6 gives the uncertainty in $T$ and $\rho$ for temperatures ranging from 300 to $700 \mathrm{~K}$, with $u_{k}=9 \mathrm{~m} / \mathrm{s}, r_{R}=6.5 \mathrm{~mm}$, and sampling rate of $10 \mathrm{kHz}$. The measurement uncertainty of all parameters increases as temperature increases. This is attributable to the lower gas density at higher temperatures, resulting in less scattering molecules, and hence fewer scattered photons. Although the uncertainty in the parameters is rather high for instantaneous measurements, long data records allow for calculation of higher accuracy statistical quantities such as power spectra and mean square fluctuations. As sampling rate decreases, so will uncertainties, since the uncertainty is inversely related to the total number of photon counts, as shown in Eqs. 15. Experimental data was acquired at sampling rates of 1 and $10 \mathrm{kHz}$. The lower sampling rate increased photon counts by a factor of 10 , thereby improving uncertainty levels by a factor of $\sqrt{10}$ over the higher sampling rate. 


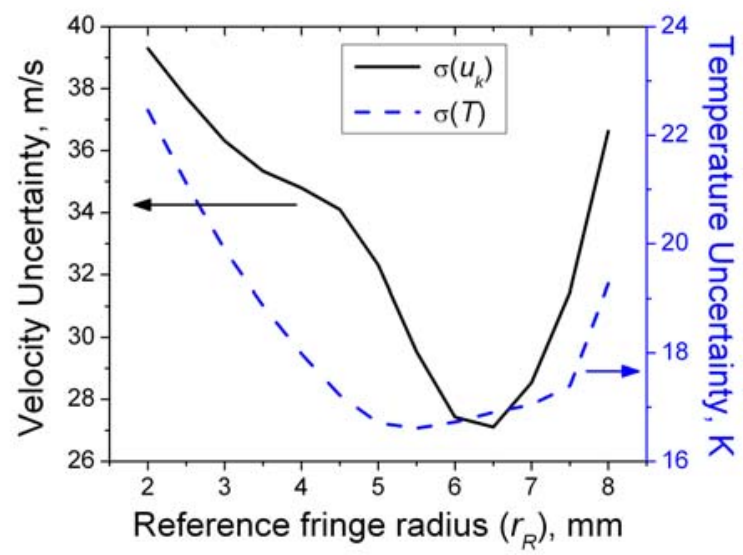

Figure 5. Uncertainty in velocity and temperature as a function of reference fringe radius; $u_{k}=9 \mathrm{~m} / \mathrm{s}$, $T=360 \mathrm{~K}$.

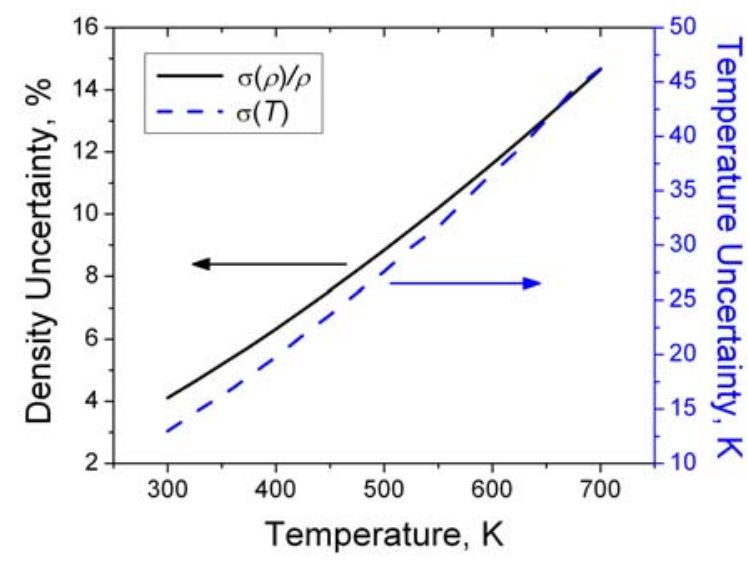

Figure 6. Uncertainty in density and temperature as a function of temperature; $u_{k}=9$ $\mathrm{m} / \mathrm{s}, r_{R}=6.5 \mathrm{~mm}$.

\section{Experiment}

Flow measurements were performed downstream of a heated air stream issuing from an $8 \mathrm{~mm}$ diameter straight tube surrounded by a $50 \mathrm{~mm}$ diameter $2 \mathrm{~m} / \mathrm{s}$ room temperature co-flow at the NASA Glenn Research Center using the described Rayleigh scattering measurement technique. The core flow was heated via electrically heated coils inside the tube, which generated turbulent mixing in the exiting air stream. The heated air jet was operated over a static temperature range of 295 to $700 \mathrm{~K}$ with a mean centerline velocity ranging from 8 to $12 \mathrm{~m} / \mathrm{s}$ measured at an axial distance of $32 \mathrm{~mm}$ ( 4 jet diameters) from the tube exit. The Reynolds number ranged from 1500 - 3000 . A PID feedback control unit was used to adjust and maintain the jet total temperature to within $+/-1.5 \mathrm{~K}$ of the set-point temperature. Since the measurement technique relies on having particulate free gas flows, a series of filters were placed in line with the air plumbing to remove dust, oil and water from both air supplies. Figure 7a shows the layout of the optics around the jet, which were used to collect Rayleigh scattered light from gas molecules in the flow. The jet was mounted such that the main flow direction was parallel to the table. A $5 \mathrm{~W}, 532 \mathrm{~nm}$ wavelength, singlefrequency, Nd:Vanadate CW laser provided incident light for the system. The laser beam was focused with a 250 $\mathrm{mm}$ focal length lens (L1) to a $70 \mu \mathrm{m} 1 / \mathrm{e}^{2}$ diameter at the probe volume positioned $32 \mathrm{~mm}$ downstream of the jet exit. The beam was oriented in the horizontal plane, at a $45^{\circ}$ angle to the primary flow direction. Light was collected at a $90^{\circ}$ scattering angle, collimated by a f/4 $200 \mathrm{~mm}$ focal length lens (L2), and focused by a $100 \mathrm{~mm}$ focal length lens (L3) onto a $0.55 \mathrm{~mm}$ diameter multimode optical fiber. Since the pair of lenses provided 1:2 imaging, the length of the probe volume was $1.1 \mathrm{~mm}$. The incident and scattering wave vectors were arranged such that the axial component of the jet velocity was measured, as shown in Fig. 7a. The jet was mounted on vertical and horizontal translation stages so that the $1.1 \mathrm{~mm}$ probe volume could be positioned anywhere in the jet plume.

The fiber was routed to a separate optical table where spectral analysis and detection took place, shown in Fig. 7b. The light exiting the fiber was collimated by an $80 \mathrm{~mm}$ focal length lens (L5) and was directed through the planar mirror FPI. The FPI had $70 \mathrm{~mm}$ diameter mirrors with $80 \%$ reflectivity, $8.7 \mathrm{GHz}$ free spectral range (FSR), and reflective finesse of approximately 16. The Fabry-Perot is an extremely sensitive instrument; even the smallest vibrations or temperature changes can cause the mirrors to drift out of parallel alignment, resulting in increased uncertainty. Therefore, a stabilization system was utilized to maintain parallelism of the mirrors during testing. Between Rayleigh scattering measurements, a mirror and diffuser (Fig. 7a) were placed in the beam path by linear actuators to direct some of the incident laser beam into the optical fiber and through the FPI. A set of reflecting prisms mounted on a linear actuator (Fig. 7b) was positioned in the optical path at the output of the interferometer to direct the light from three regions of the interferometer mirrors to a video camera. Live video of the three fringe images was used in a feedback control loop to adjust the mirror positions using piezoelectric transducers until the three fringes were equal in diameter. This system was also used to set the fringe diameter of the incident reference light to $13 \mathrm{~mm}$, as determined from the uncertainty analysis. Further details regarding the mirror stabilization system for the FPI can be found in Ref. 17.

When flow measurements were collected the prisms, mirror, and diffuser were removed from the optical path and the light exiting the FPI was focused by a fringe forming lens (L6) having an effective focal length of $2584 \mathrm{~mm}$, 


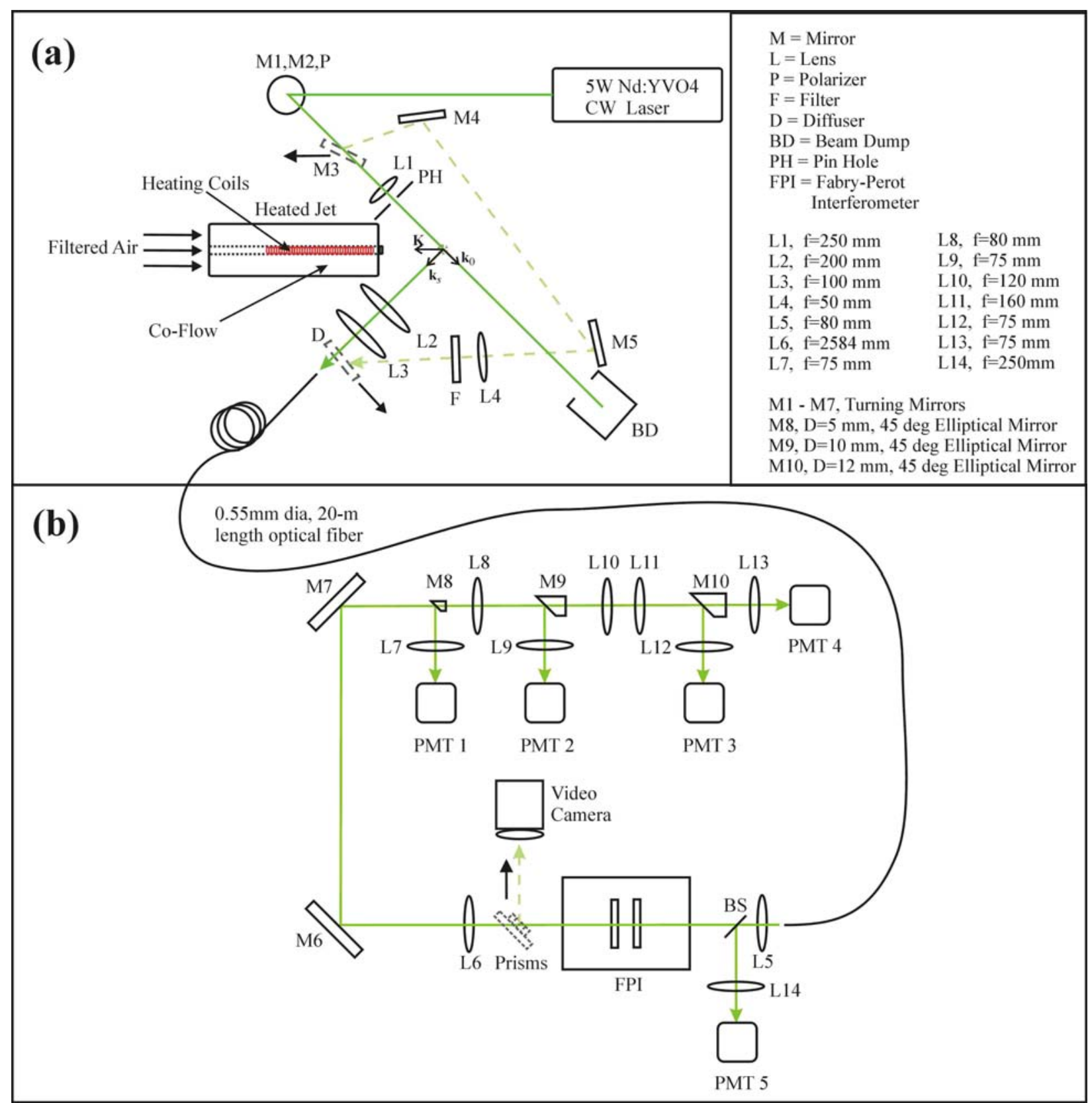

Figure 7. Diagram of experiment setup: (a) laser and collection optics; (b) spectral analysis and detection equipment.

which provided an $18 \mathrm{~mm}$ diameter image of the fiber face. The light was sampled via a series of various diameter $45^{\circ}$ elliptical mirrors (Fig. 7b). Mirror M8 sent the central $5 \mathrm{~mm}$ diameter section of the fringe image to PMT 1. Lens L8 refocused the remaining portion of the image with a magnification factor of 0.87 at mirror M9 which sent the central $11.5 \mathrm{~mm}$ diameter section of the original image to PMT 2. A pair of lenses (L10 and L11) refocused and de-magnified the image again so that mirror M10 removed a circular section equivalent to the central $16.1 \mathrm{~mm}$ diameter region of the original image, and sent this light to PMT 3. The remaining outermost annular region of the image was focused at PMT 4. Figures 8 and 9 illustrate the individual areas of the fringe image which are directed towards each PMT. The PMTs were operated in the photon counting mode to acquire fringe intensity data. The photoelectron pulses from the five PMTs were amplified (Gain =5) and sent to photon counters, which output NIM level pulses. Constant fraction discriminators converted the NIM pulses to 10 ns wide TTL level pulses that were counted via counter-timer boards. Typical photoelectron count rates for this work were on the order of $1 \mathrm{MHz}$. A 


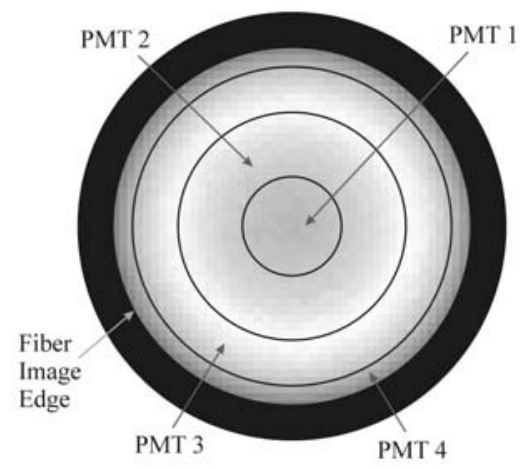

Figure 8. Dissection of Fabry-Perot fringe pattern into four annular regions.

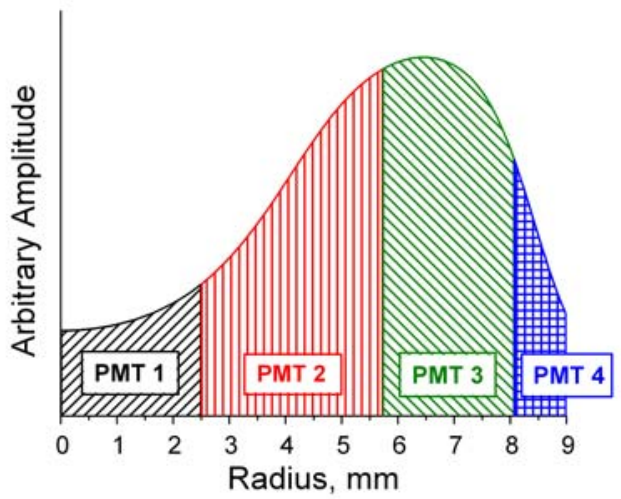

Figure 9. Radial slice of a fringe pattern showing the annular regions detected by the four PMTs. The amount of light in each region changes as the velocity and temperature fluctuate.

PC-based data acquisition system was used to record the signals from the PMTs. Each signal channel was digitized at either 1 or $10 \mathrm{kHz}$ sampling rate for 30 seconds, resulting in 30,000 or 300,000 samples per channel. The intensity information from the Fabry-Perot fringe sampling was used to obtain temperature and velocity measurements. The density measurement was much simpler, requiring only an overall intensity measurement. A beamsplitter located just in front of the FPI input (Fig. 7b) directed approximately 10\% of the incoming Rayleigh scattered light to a lens (L14), which focused the light at PMT 5 for density measurement.

The temperature, density, and velocity measurements at the probe location were verified using additional instrumentation. A fine-wire $(0.25 \mathrm{~mm}$ diameter $)$ open-bead type $\mathrm{K}$ thermocouple provided jet static temperature data. A pressure gauge measured the ambient (static) pressure. Gas density information was derived using the ideal gas law. The jet velocity profile was evaluated from total pressure measurements with a pitot probe. A constant current anemometry (CCA) system provided static temperature fluctuation measurements. The system consisted of a $1.3 \mu \mathrm{m}$ diameter, $0.9 \mathrm{~mm}$ long platinum resistance wire operated in a constant current configuration. The frequency response of the CCA system, based on the $3 \mathrm{~dB}$ point, was measured to be approximately $1 \mathrm{kHz}$.

\section{Results}

\section{A. Calibration and technique verification}

The technique was calibrated over a jet static temperature range of $295-700 \mathrm{~K}$ and velocity range of $8-12 \mathrm{~m} / \mathrm{s}$. Thermocouple and pitot probe measurements were used to determine the mean temperature and velocity of the gas at the center of the jet plume $32 \mathrm{~mm}$ downstream of the tube exit. Since thermocouples measure the temperature of the metal probe bead rather than the gas temperature, there may be a difference between the actual gas temperature and the thermocouple measurement. These differences result from conduction losses down the thermocouple wire and radiation losses to the surroundings. The error in thermocouple-measured gas temperature was estimated to be less than $\pm 3 \mathrm{~K}$ for the temperatures studied here. Gas density was derived from thermocouple and pressure probe measurements. The photoelectron counts recorded by PMT 5 are linearly related to density as shown by the calibration curve in Fig. 10 for data acquired at two sampling rates ( 1 and $10 \mathrm{kHz})$. The photon counts were adjusted by a small amount to account for stray laser light and provide an intercept that indicates nearly zero photon counts at zero density. 


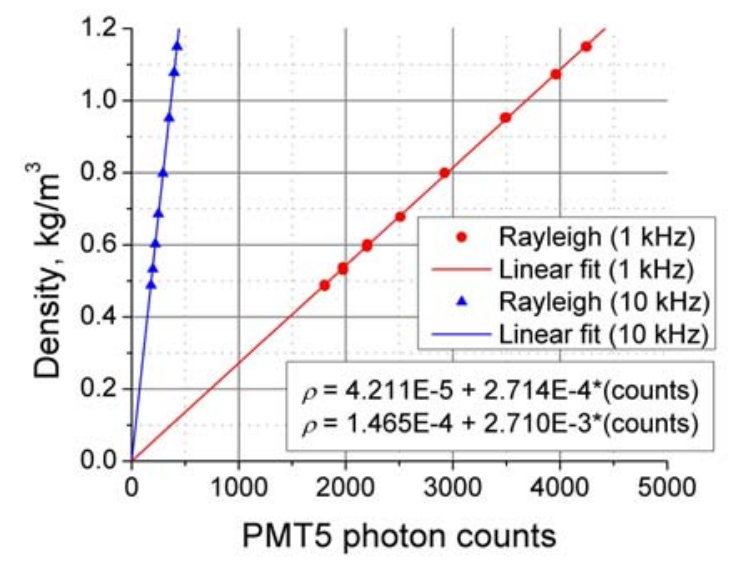

Figure 10. Density as a function of PMT 5 photon counts.

The axial velocity component and temperature were measured simultaneously by analyzing the light from four annular regions of the interference fringe pattern. A model of the fringe pattern, including the Rayleigh scattering spectrum and FPI instrument function, was used in a least squares fitting routine to estimate unknown parameters from the intensity data. Mean temperature, velocity, and pressure measured by physical probes were used as known values in the least squares analysis of the mean photon count data at the calibration points, while several unknown factors were evaluated over the range of temperatures and velocities. These unknown factors included system detection efficiencies, effective finesse of the FPI, and fringe forming lens focal length. Once these factors were calibrated, the mean PMT counts acquired at sampling rates of 1 and $10 \mathrm{kHz}$ were analyzed to evaluate the mean temperature and velocity at each data point, with the constraint that the pressure was equal to the ambient value measured by the pressure probe. Figures 11 and 12 respectively show thermocouple and pitot probe measurements compared with temperature and velocity measurements from the Rayleigh technique over the range of calibration temperatures. The temperature measurements agree extremely well for the $1 \mathrm{kHz}$ sampling rate, but start to deviate at the high end of the temperature range for the $10 \mathrm{kHz}$ sampling rate due to extremely low photon counts. The velocity measurements appear quite poor at a first glance at Fig. 12. Keep in mind that the measurement uncertainty is on the order $10 \mathrm{~m} / \mathrm{s}$, which is approximately the same magnitude as the velocity being measured, so the relative uncertainty is very high. Error bars have been shown to represent the standard deviation of the instantaneous measurements. At higher velocities $(>100 \mathrm{~m} / \mathrm{s}), 10 \mathrm{~m} / \mathrm{s}$ uncertainty is not as significant. Although the velocity uncertainty is relatively high for the low velocities studied here, the results are shown to verify that simultaneous measurement of temperature and velocity is feasible.

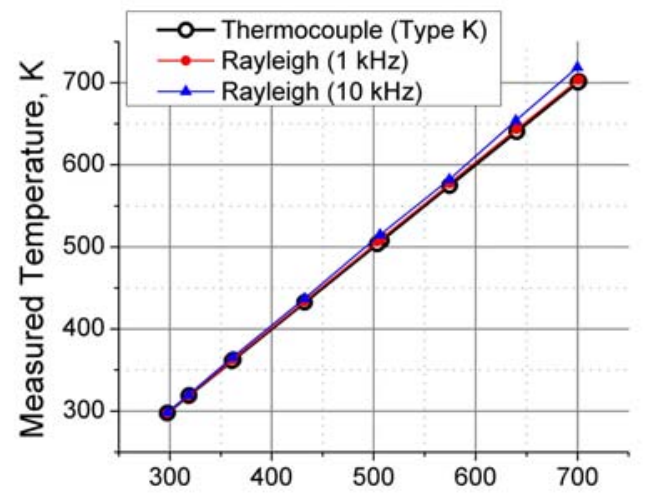

Thermocouple-measured Temperature, $\mathrm{K}$

Figure 11. Mean temperature measurements from Rayleigh technique compared with thermocouple measurements.

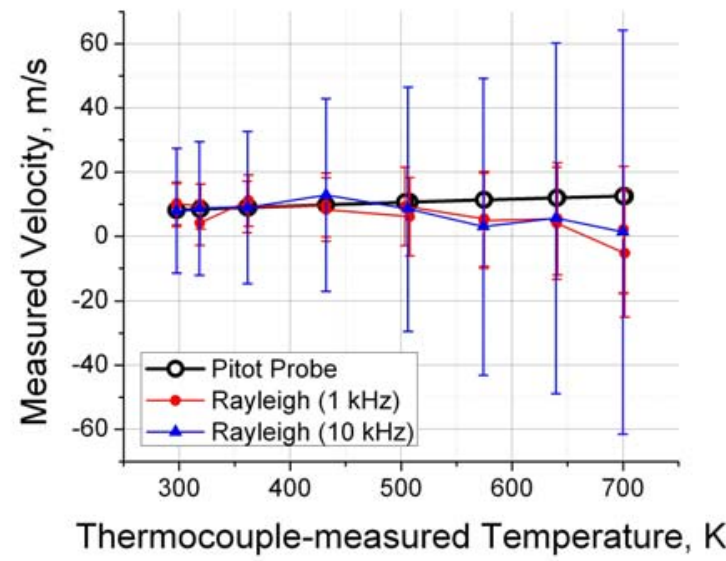

Figure 12. Mean velocity measurements from Rayleigh technique compared with pitot probe measurements. The error bars represent the standard deviation of the instantaneous Rayleigh-measured velocity values. 
Using the linear equation relating PMT 5 photon counts to density (Fig. 10), and least squares analysis of the photon counts from PMTs 1-4, time histories of instantaneous density, temperature, and velocity were evaluated at each data point. The power spectra and mean square fluctuations were calculated from this information. Figures 13 and 14 respectively show the first 5 seconds of the time history temperature results from the CCA measurements and the Rayleigh measurements acquired at $1 \mathrm{kHz}$ sampling rate. The 2 second cycles in the data were due to the temperature controller time setting of one second on, one second off. The Rayleigh data demonstrates higher noise levels due to shot noise in the PMT signals; however the results verify that the Rayleigh temperature measurements sufficiently reproduce the CCA results.

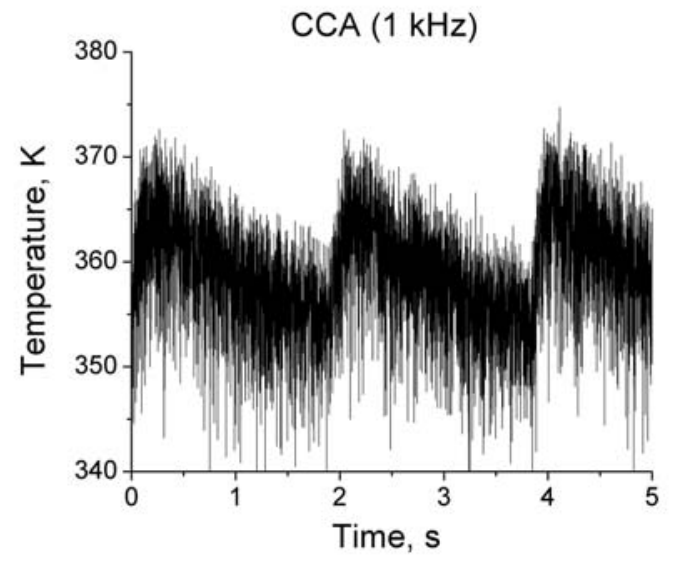

Figure 13. Time history of instantaneous CCA temperature measurements.

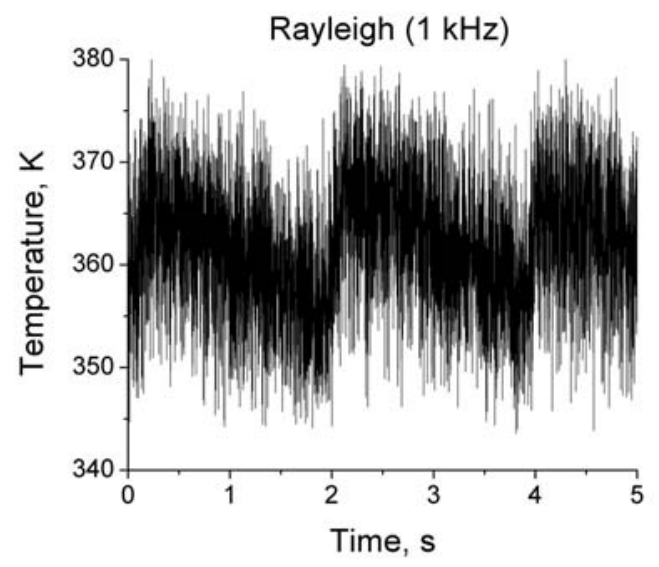

Figure 14. Time history of instantaneous Rayleigh temperature measurements.

\section{B. Power Spectral Density (PSD) calculations}

The Rayleigh probe volume and the CCA probe were scanned vertically across the jet centerline at an axial station of $32 \mathrm{~mm}$. Scans were performed at centerline jet conditions of $360 \mathrm{~K}$ and $9 \mathrm{~m} / \mathrm{s}$. These radial scans provided measurements in the jet core as well as in the turbulent mixing layer formed between hot and cold air streams. Because of the shot noise in the PMT signals, it was necessary to use relatively long data records and calculate power spectral densities using a technique such as the Welch method of modified periodograms ${ }^{25}$. Due to extensive data processing time (approximately 700 samples $/ \mathrm{min}$ ), only the first 6 seconds of the $10 \mathrm{kHz}$ data were analyzed. In the Welch method, a data record sampled at a rate $f_{s}=10 \mathrm{kHz}(1 \mathrm{kHz})$ for a total time of $6 \mathrm{sec}(30 \mathrm{sec})$ was subdivided into smaller records of length $L=1024$ (128) samples, which were overlapped by $50 \%$. The modified periodograms of each sub-record were calculated using a data window. These individual periodograms were then averaged to obtain the estimate of the power spectrum. The frequency resolution of the resulting spectrum is $f_{s} / L=$ $9.77 \mathrm{~Hz}(7.81 \mathrm{~Hz})$. Overlapping the segments by $50 \%$ provided a near maximum reduction in the variance in the spectral estimate. The resulting power spectral densities provide fluctuation information out to $5 \mathrm{kHz}(0.5 \mathrm{kHz})$. The sum of all points in the PSD is equivalent to the mean square fluctuations.

Figures 15-17 show temperature PSD plots for three radial measurement locations: centerline $\left(r_{j}=0\right)$, shear layer $\left(r_{j}=6 \mathrm{~mm}\right)$, and a region outside the mixing layer $\left(r_{j}=9 \mathrm{~mm}\right)$, calculated from the $1 \mathrm{kHz}$ CCA data and the 1 and $10 \mathrm{kHz}$ Rayleigh data. The shot noise results in a constant noise floor in the spectra. This noise floor was apparent in the $10 \mathrm{kHz}$ data at frequencies above $2.5 \mathrm{kHz}$ where the spectrum is very flat (no fluctuations). The noise floor could not be estimated from the $1 \mathrm{kHz}$ data since the power spectrum does not provide information at higher frequencies where it is safe to say that there is no contribution from true temperature fluctuations. Therefore, the shot noise contribution has only been subtracted from the $10 \mathrm{kHz}$ spectra. Comparison between the temperature PSD results obtained from the Rayleigh data and the CCA data in the $0-0.5 \mathrm{kHz}$ range indicates that the shape of the spectrum is accurately calculated from both Rayleigh data sets; however, slight amplitude differences do exist. The $1 \mathrm{kHz}$ Rayleigh data still contains the shot noise contribution, resulting in a constant offset in amplitude from the CCA data, which is most apparent in figs. 15 and 16 . The $10 \mathrm{kHz}$ spectra were not calculated with the same frequency resolution as the $1 \mathrm{kHz}$ spectra since the length of segments $(L)$ must be a power of 2 and the sampling rates $\left(f_{s}\right)$ varied by a factor of 10 . The frequency "bins" for the $10 \mathrm{kHz}$ spectra are nearly $2 \mathrm{~Hz}$ larger, resulting in slightly more "power" at each point in the spectrum (Fig. 16). As expected, the strongest fluctuations are found in the shear 
layer, where the higher temperature air from the jet core is mixing with the colder co-flow air. The spectra at the other two locations are relatively flat.

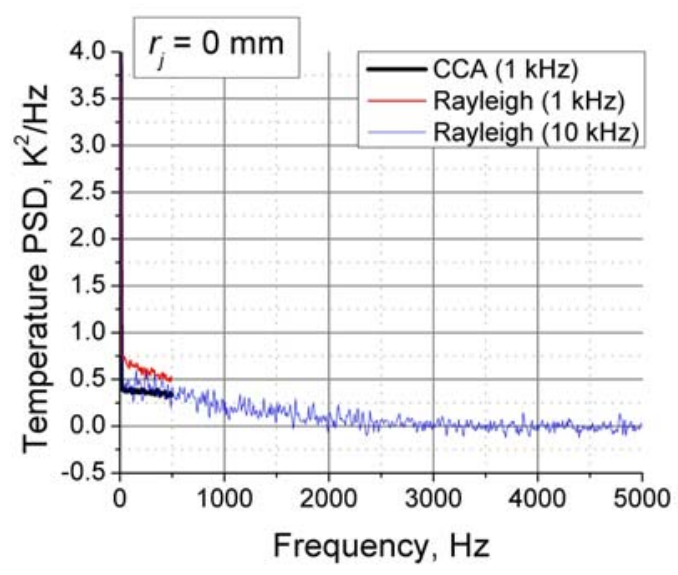

Figure 15. Power spectral density of temperature fluctuations at jet centerline.

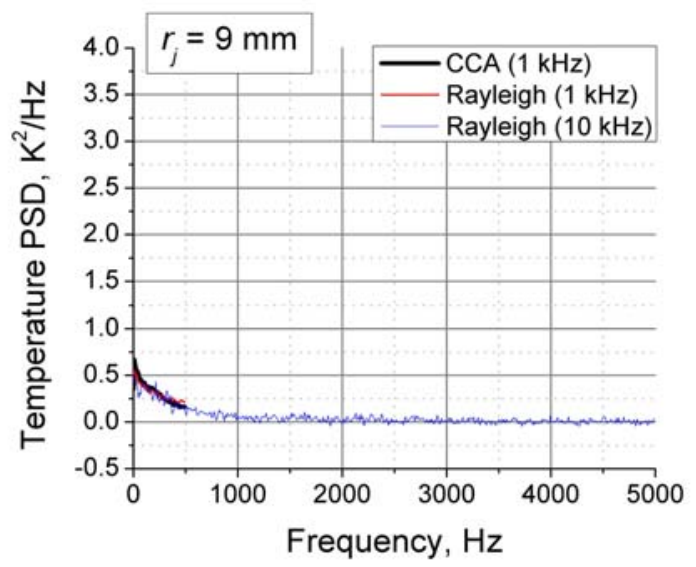

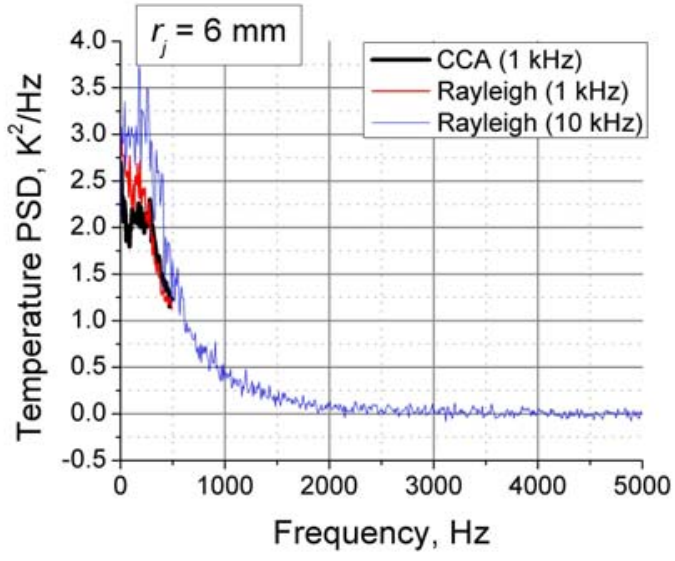

Figure 16. Power spectral density of temperature fluctuations in jet shear layer.

\section{Figure 17. Power spectral density of temperature fluctuations in co-flow region.}

Figures 18 and 19 show sample density and velocity PSD plots, respectively. The density PSD is shown for a location in the shear layer demonstrating strong density fluctuations resulting from strong temperature fluctuations. The velocity spectra at all locations were very similar and the centerline location was chosen as a typical case. The frequency scale has only been shown to $2.5 \mathrm{kHz}$ to show finer detail in the low frequency range. Velocity fluctuations were very small due to the low speed of the jet; however, dominant fluctuations are seen at 48 and 430 $\mathrm{Hz}$, indicated by large spikes in the $10 \mathrm{kHz}$ velocity PSD. The jet velocity does not actually have strong fluctuations at these frequencies. Rather, these spikes in the spectra can be explained by external noise sources that affect the frequency of the incident light. Since the velocity measurement is based on a shift in frequency from the incident laser frequency, fluctuations in the laser frequency become manifest in the velocity measurements. The $430 \mathrm{~Hz}$ spike was seen in previous Rayleigh measurement work in a free jet ${ }^{17}$ using the same laser model, suggesting that the laser has a resonance mode around $430 \mathrm{~Hz}$ that is excited by the broadband jet noise. The $430 \mathrm{~Hz}$ modulation was reduced in the previous work by mounting the laser head inside an anechoic box. Modulation induced by a small motor used to remove speckle from the light transmitted by the fiber, often referred to as "scrambling", may explain the $48 \mathrm{~Hz}$ spike. 


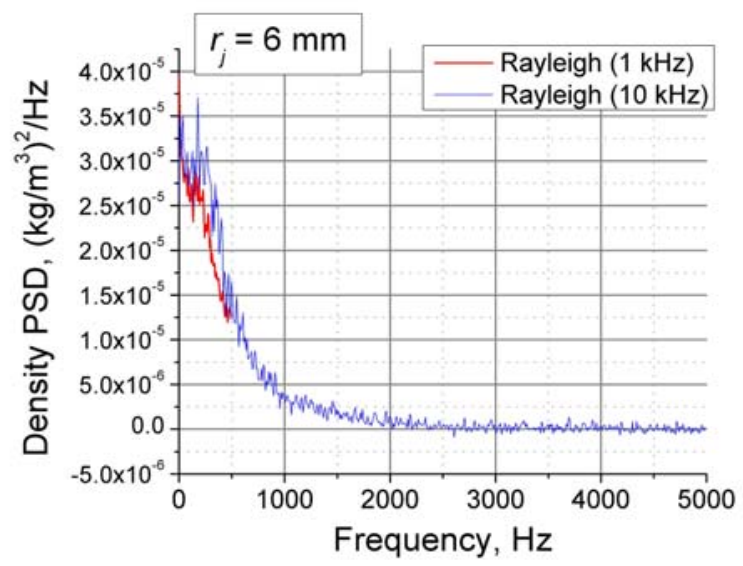

Figure 18. Power spectral density of density fluctuations in jet shear layer.

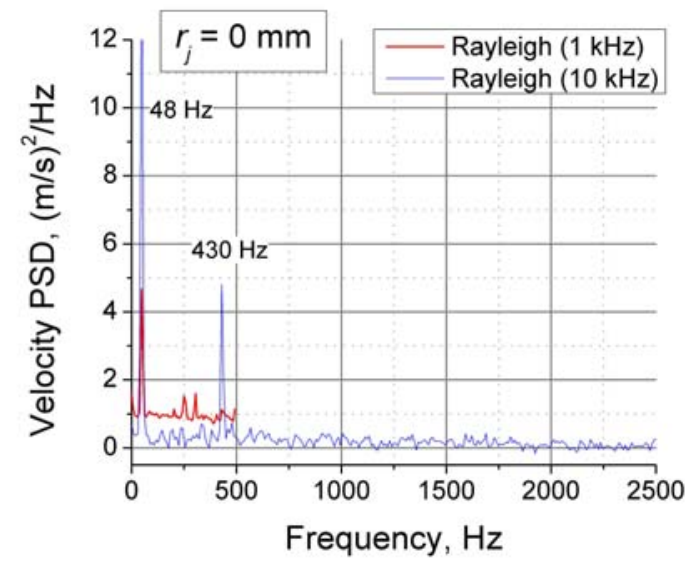

Figure 19. Power spectral density of velocity fluctuations at jet centerline.

\section{Jet profiles}

The mean temperature and velocity were calculated from the time history data, and the turbulence temperature and velocity fluctuations were derived from the power spectral density calculations by taking the sum of all points in the PSD. Figures 20 and 21 show profiles resulting from radial scans across the jet flow of the mean temperature and turbulent temperature fluctuations calculated from the $1 \mathrm{kHz}$ CCA measurements and the 1 and $10 \mathrm{kHz}$ Rayleigh measurements. Mean temperature values from the three data sets are found to be within $+/-3 \mathrm{~K}$ of each other. The CCA measurements provide very accurate turbulence levels for fluctuations up to $0.5 \mathrm{kHz}$; however, as the $10 \mathrm{kHz}$ power spectra show (figs. 15-17), significant temperature fluctuations still exist beyond $0.5 \mathrm{kHz}$. Since the $1 \mathrm{kHz}$ CCA and Rayleigh measurements do not account for higher frequency fluctuations, the resulting turbulence levels are lower than those calculated from $10 \mathrm{kHz}$ Rayleigh data. The shot noise contribution was not removed from the 1 $\mathrm{kHz}$ Rayleigh data, resulting in slightly higher fluctuation measurements than the CCA data indicates in regions where temperature fluctuations are small and shot noise dominates (i.e., in the jet core and in the co-flow).

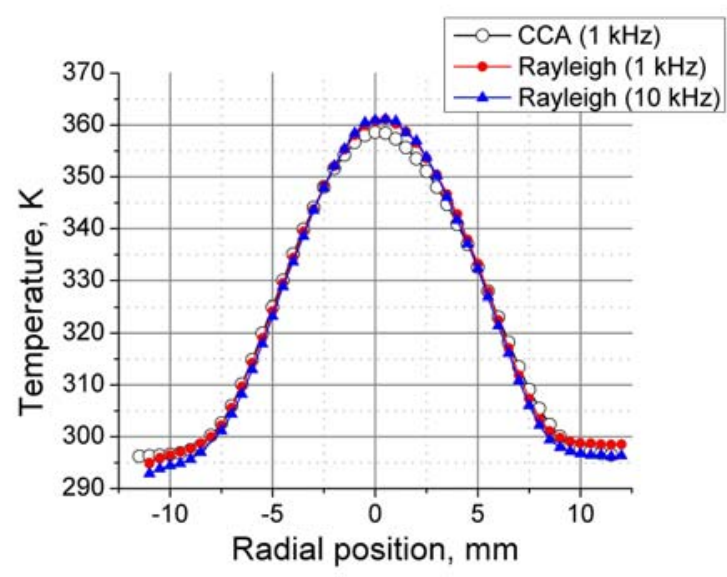

Figure 20. Mean temperature profile at an axial station $32 \mathrm{~mm}$ downstream of the tube exit.

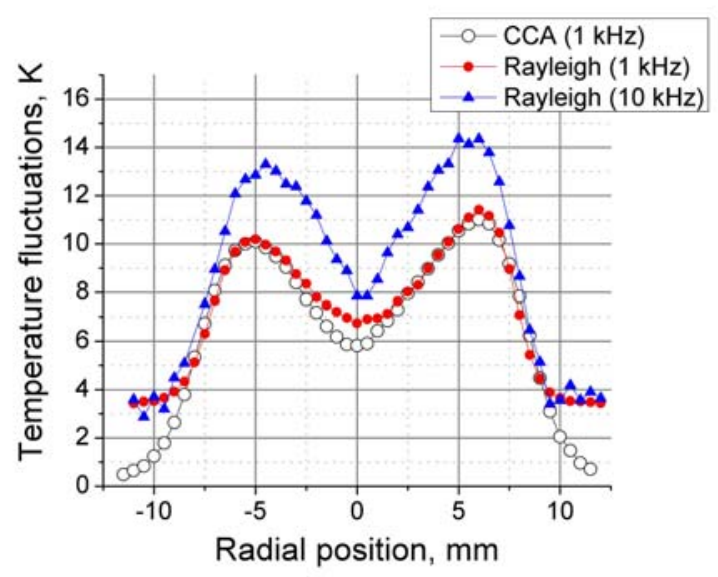

Figure 21. Temperature fluctuation profile at an axial station $32 \mathrm{~mm}$ downstream of the tube exit.

Figure 22 shows profiles of the mean velocity measured by the pitot probe and calculated from the 1 and $10 \mathrm{kHz}$ Rayleigh data. As mentioned in section IV.A., the relative error in the velocity measurements is quite high since the flow velocity is so low. The error in velocity is about $+/-5 \mathrm{~m} / \mathrm{s}$ while the maximum velocity is only $9 \mathrm{~m} / \mathrm{s}$. The results show that it is feasible to obtain velocity measurements with the presented Rayleigh technique; however the measurements are more applicable in higher velocity flows where the relative error is much lower. It appears from Fig. 22 that the velocity measurements on the lower side of the jet flow are much noisier than those obtained above 
the jet centerline. This may be due to differences in the amount of stray light entering the optical fiber as the jet is translated. The amount of stray light was found to be greater for negative probe locations than for positive ones. The stray light level has been evaluated and accounted for in the density calculations from PMT 5. The amount of light is not accounted for in the least squares analysis of PMT 1-4 data since it did not seem to have a significant affect on the results during the calibration study. In the future, more precautions for eliminating stray light will be taken to improve the measurements. Figure 23 shows the turbulent velocity fluctuations calculated from the $1 \mathrm{and} 10 \mathrm{kHz}$ Rayleigh measurements. The actual velocity fluctuations in this jet are unknown since hotwire velocity measurements were not available for comparison. The turbulence levels shown here are higher than expected since the maximum fluctuations in jets are typically $15-20 \%$ of the difference between the centerline velocity and ambient or co-flow velocity. For this flow, maximum fluctuations should be on the order of $1 \mathrm{~m} / \mathrm{s}$. Sources of error in the velocity turbulence levels calculated from the Rayleigh data may include fluctuations induced by laser frequency modulation, shot noise contribution, and noise from stray laser light.

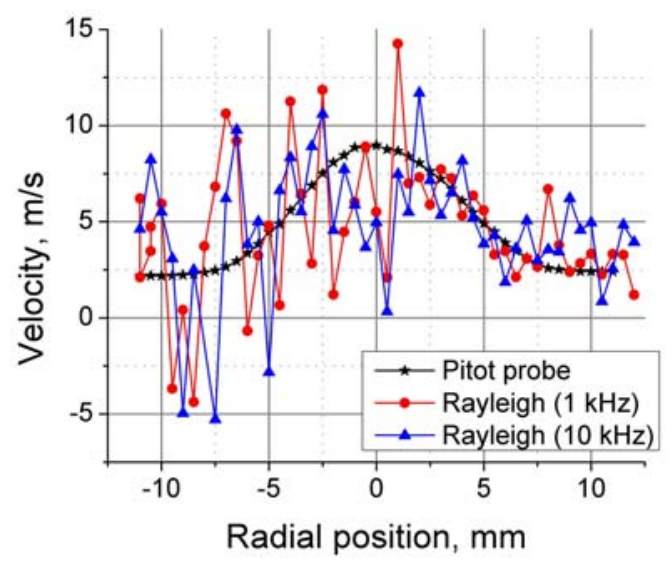

Figure 22. Mean velocity profile at an axial station $32 \mathrm{~mm}$ downstream of the tube exit.

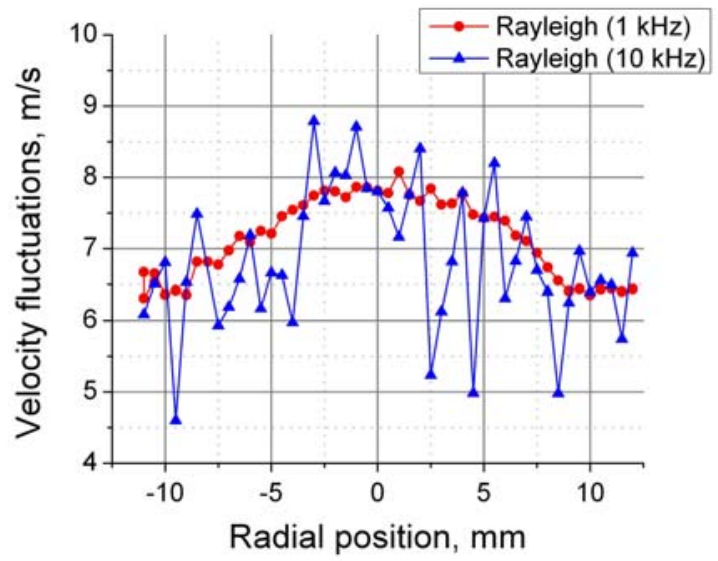

Figure 23. Velocity fluctuation profile at an axial station $32 \mathrm{~mm}$ downstream of the tube exit.

\section{Conclusion}

A technique for obtaining dynamic gas temperature, velocity, and density measurements using molecular Rayleigh scattering was described. Density was determined from an overall intensity measurement of the scattered light, while temperature and velocity were determined by analyzing the scattered light with a Fabry-Perot interferometer. The signals from five photomultiplier tubes were simultaneously recorded using photon counting electronics operating at 1 and $10 \mathrm{kHz}$ sampling rates with 30 second recording periods. An uncertainty analysis was presented that demonstrated uncertainties in instantaneous temperature, velocity, and density measurements of about $17 \mathrm{~K}, 27 \mathrm{~m} / \mathrm{s}$, and $4 \%$, respectively, for a $10 \mathrm{kHz}$ sampling rate. Derived statistical quantities, such as mean square fluctuations and power spectra, can be obtained with high accuracy if inherent noise contributions are eliminated, either experimentally or numerically in the data processing. Measurements using the Rayleigh technique were demonstrated in a low speed heated air flow and were verified by comparing results with CCA and pitot probe measurements.

In future work, a higher power laser will be used to increase photon counts, thereby improving measurement uncertainty. Measurements will be made in a high speed heated flow where velocity fluctuations are significant, and fluctuations will be verified by hotwire velocity measurements. The presented Rayleigh scattering technique will eventually be used in aeroacoustics research, where sound pressure fluctuation (microphone) measurements will be acquired simultaneously with Rayleigh measurements allowing correlation between flow property fluctuations and noise generation. Determining sources of jet noise will help engineers to design quieter, more efficient aircrafts.

\section{Acknowledgments}

We would like to thank Dick Seasholtz, Jay Panda, and Mark Wernet for their technical advice. 


\section{References}

${ }^{1}$ Eckbreth, A. C., Laser Diagnostics for Combustion Temperature and Species, Gordon and Breach Science Publishers SA, Amsterdam, 1996, pp.209-451.

${ }^{2}$ Cummings, E. B., "Laser-Induced Thermal Acoustics," Ph.D. Dissertation, Department of Engineering and Applied Science, California Institute of Technology, Pasadena, CA, 1995.

${ }^{3}$ Hart, R. C., Balla, R. J., and Herring, G. C., "Nonresonant referenced laser-induced acoustics thermometry in air," Appl Optics, Vol. 38, No. 3, 1999, pp. 577-584.

${ }^{4} \mathrm{Li}$, Y., Roberts, W. L., and Brown, M. S., "Investigation of gaseous acoustic damping rates by Transient Grating Spectroscopy," AIAA Journal, Vol. 40, No. 6, 2002, pp. 1071-1077.

${ }^{5}$ Lau, J. C., Morris, P. J., and Fisher, M. J., "Measurements in subsonic and supersonic free jets using a laser velocimeter," $J$ Fluid Mech, Vol. 93, Jul, 1979, pp. 1-27.

${ }^{6}$ Lau, J. C., Whiffen, M. C., Fisher, M. J., and Smith, D. M., “A note on turbulence measurements with a laser velocimeter,” $J$ Fluid Mech, Vol. 102, Jan, 1981, pp. 353-366.

${ }^{7}$ Flack, R. D., "Influence of turbulence scale and structure on individual realization laser velocimeter biases," J Phys E: Sci Instrum, Vol. 15, No. 10, 1982, pp. 1038-1044.

${ }^{8}$ Heist, D. K., Castro, I. P., "Point measurement of turbulence quantities in separated flows-a comparison of techniques," Meas Sci Technol, Vol. 7, No. 10, 1996, pp. 1444-1450.

${ }^{9}$ Elliott, G. S., and Boguszki, M., "Filtered Rayleigh scattering: toward multiple property measurements," AIAA-2001-0301, 2001.

${ }^{10}$ Bridges, J., Wernet, M. P., and Brown, C., “Control of jet noise through mixing enhancement,” NASA TM-212335, 2003.

${ }^{11}$ Garg, S., and Settles, G. S., "Measurements of a supersonic turbulent boundary layer by focusing schlieren deflectometry,"Exp Fluids, Vol. 25, No. 3, 1998, pp. 254-264.

${ }^{12}$ Seasholtz, R. G., and Greer, L. C., "Rayleigh scattering diagnostic for measurement of temperature and velocity in harsh environments," AIAA-98-0206, 1998.

${ }^{13}$ Panda, J., and Seasholtz, R. G., "Velocity and temperature measurement in supersonic free jets using spectrally resolved Rayleigh scattering," AIAA-99-0296, 1999.

${ }^{14}$ Mielke, A. F., Seasholtz, R. G., Elam, K. A., and Panda, J., "Time-average measurement of velocity, density, temperature, and turbulence velocity fluctuations using Rayleigh and Mie scattering," Exp Fluids, Vol. 39, No. 2, 2005, 441-454.

${ }^{15}$ Lock, J. A., Seasholtz, R. G., and John, W. T., "Rayleigh-Brillouin scattering to determine one-dimensional temperature and number density profiles of a gas flow field," Appl Optics, Vol. 31, No. 15, 1992, pp. 2839-2848.

${ }^{16}$ Seasholtz, R. G., and Panda, J., "Rayleigh scattering diagnostic for dynamic measurement of velocity and temperature," AIAA-99-0641, 1999.

${ }^{17}$ Seasholtz, R. G., Panda, J., and Elam, K. A., "Rayleigh scattering diagnostic for measurement of velocity and density fluctuation spectra," AIAA-2002-0827, 2002.

${ }^{18}$ Mielke, A. F., and Elam, K. A., "Molecular Rayleigh scattering diagnostic for measurement of high frequency temperature fluctuations," Proceedings of SPIE Optical Diagnostics Conference, Vol. 5880, The International Society of Optical Engineering, Bellingham, WA, 2005, pp. K1-K12.

${ }^{19}$ Tenti, G., Boley, C., D., and Desai, R. C., "Velocity and temperature measurement in supersonic free jets using spectrally resolved Rayleigh scattering," Can J Phys, Vol. 52, No. 4, 1974, pp. 285-290.

${ }^{20}$ Sandoval, R. P., and Armstrong, R. L., "Rayleigh-Brillouin spectra in molecular nitrogen," Phys Rev A, Vol. 13, No. 2, 1976, pp. 752-757.

${ }^{21}$ Yip, S., and Nelkin, M., "Application of a kinetic model to time-dependent density correlations in fluids," Phys Rev, Vol. 135, No. 5A, 1964, pp. A1241-A1247.

${ }^{22}$ Boley, C. D., Desai, R. C., and Tenti, G., "Kinetic models and Brillouin scattering in a molecular gas," Can J Phys, Vol. 50, No. 18,1972 , pp. 2158-2173.

${ }^{23}$ Vaughan, J. M., The Fabry Perot Interferometer, History, Theory, Practice, and Applications, Adam Hilger, Philadelphia, 1989, pp. 89-134.

${ }^{24}$ Whalen, A. D., Detection of Signals in Noise, Academic Press, New York, 1971, pp. 327-332.

${ }^{25}$ Welch, P. D., "The use of fast Fourier transform for the estimation of power spectra: A method based on time averaging over short, modified periodograms," IEEE Trans on Audio and Electroacoustics, Vol. AU-15, No. 2, 1967, pp. 70-73. 